\title{
Extensión del Bosque Nublado y su Contribución de la Lluvia Horizontal a la Precipitación Total en la Reserva Biológica Uyuca, Honduras
}

\author{
Nelson Agudelo ${ }^{1}$, José Manuel Mora ${ }^{2}$, Samantha Pérard ${ }^{3}$ y Julio César Jut Solórzano ${ }^{4}$
}

Resumen. Se ha asumido que la zona impactada por las nubes y las neblinas en el cerro Uyuca está a partir de los $1,700 \mathrm{msnm}$ y cubre un área de 237.1 ha. De éstas, el bosque latifoliado maduro multiestratificado cubre 52 ha mientras que las otras 185 ha están cubiertas por el bosque secundario conformado por rodales puros y mixtos de Pinus maximinoi y bosque latifoliado. Ante la importancia de los bosques nublados o nubosos como recolectores de agua se cuantificó la precipitación horizontal u oculta a nivel de la masa latifoliada madura durante cinco años (1987-1992). Para ello se distribuyó una red de 12 pluviómetros tipo embudo, de 52 cm de diámetro, a varias altitudes dentro del bosque. Como control se colocaron tres pluviómetros en un área abierta de 2.7 ha adyacente al bosque maduro. Se hicieron registros diarios de la precipitación, la nubosidad y la visibilidad, la dirección y la velocidad del viento a lo largo de los cinco años del estudio. La precipitación media mensual varió entre los $62.3 \mathrm{~mm}$ y los $276.3 \mathrm{~mm}$. El promedio aritmético de la precipitación total anual dentro del bosque fue de $2,021.6 \mathrm{~mm}$. No obstante, este promedio fue de 2,360 $\mathrm{mm}$ estimado mediante el método de los polígonos de Thiessen. A campo abierto se registró un promedio total anual de $978 \mathrm{~mm}$. Consecuentemente, la precipitación horizontal fue de $1,382.2 \mathrm{~mm}$, un aporte del bosque de $141.3 \%$ a la precipitación normal. Se determinó que la zona de vida del bosque muy húmedo montano bajo subtropical en el cerro Uyuca comienza a partir de los 1,600 msnm. Como resultado, el área de bosque nuboso en el cerro Uyuca es de 556 ha. Este sector adicional (322 ha), en la actualidad, no es parte del núcleo de la Reserva Biológica del Cerro Uyuca. Consecuentemente, este sector adicional o al menos sus partes más factibles deben ser estrictamente preservadas para proteger un área de bosque nublado de gran relevancia. Esto es fundamental sobre todo si se tiene en cuenta que debido a su delicada dependencia del clima local, los bosques nubosos serán fuertemente afectados por el cambio climático global. La inclusión en la zonificación de la reserva de una Subzona de Adaptación al Cambio Climático es un primer paso excelente para trabajar en contrarrestar los impactos del cambio climático y el efecto compuesto de este con otros factores de amenaza a la biodiversidad.

Palabras clave: Bosque secundario, bosque muy húmedo montano bajo subtropical, cambio climático global, precipitación normal, precipitación oculta, servicios ecosistémicos.

\section{Cloud Forest Extension and its Contribution of Occult Rain to the Total Precipitation in the Uyuca Biological Reserve, Honduras}

Abstract. It has been assumed that the zone impacted by clouds and mists on the Uyuca Mountain begins at $1,700 \mathrm{~m}$ for an area of 237 ha. Mature multistratified broadleaf forest covers 52 ha whereas 185 ha are covered by secondary forest formed by pure and mixed groves of Pinus maximinoi and broadleaf forests. Due to the importance of cloud forests as water collectors, occult precipitation o fog dip was quantified at the mature broadleaf forest over five years (1987-1992). A network of 12 funnel type pluviometers of $52 \mathrm{~cm}$ of diameter was installed at different altitudes inside the forest. Three pluviometers were placed as control in a 2.7 ha open area adjacent to the mature forest. Data were taken daily about rainfall, cloudiness, visibility, wind speed and direction, throughout the five years of the study. Monthly average rainfall varied between $62.3 \mathrm{~mm}$ and $276.3 \mathrm{~mm}$. Average total annual rainfall inside the forest was $2,021.6 \mathrm{~mm}$, whereas it was $2,360 \mathrm{~mm}$ estimated by the Thiessen's

\footnotetext{
${ }^{1}$ Medellín, Colombia. Correo electrónico nagudeloci@gmail.com

${ }^{2}$ Instituto Internacional en Conservación y Manejo de Vida Silvestre (ICOMVIS), Universidad Nacional (UNA), Heredia, Costa Rica. Correo electrónico josemora07@gmail.com

${ }^{3}$ Avenida Lázaro Cárdenas, \#18, ejido Nuevo León, Mexicali, Baja California, México. Correo electrónico perards19@gmail.com

${ }^{4}$ Rua Mustafa Saleh Abdo Sater. No. 24 Parque Alvorada, Dourados MS., Brasil. Correo electrónico julio.jut@gmail.com
} 
polygons method. A total annual average of 978 was registered at the open area. Consequently horizontal rainfall was $1,382.2 \mathrm{~mm}$; a $141.3 \%$ contribution of the forest to the normal rainfall. It was determined that the very humid mountainous low subtropical forest zone begins at 1,600 msnm in Uyuca. As a result, the area covered by cloud forest at the Uyuca mountain measures 556 ha. Consequently, this additional sector, or at least the most feasible parts of it, must be strictly preserved to protect an area of cloud forest of great importance. This is essential especially if we take in account that due to its delicate dependence on local climate, cloud forests will be strongly affected by global climate change. The inclusion on the reserve zoning of a Climate Change Adaptation Subzone is an excellent first step in working to counteract the impacts of climate change and the effect of this compounded with other factors that threat biodiversity.

Key words: Ecosystem services, fog dip, global climate change, mature forest, secondary forest, very humid mountainous low subtropical forest.

\section{Introducción}

En las porciones más altas y frías de los sistemas montañosos se encuentran los bosques de niebla 0 neblina, también conocidos como bosques nubosos o nublados. Por lo general, estos ecosistemas están localizados en las porciones más altas de las montañas en las regiones tropicales y subtropicales. Los bosques nublados en la región templada pueden estar ubicados próximos al nivel del mar (Stadtmüller 1987). En general, los bosques nublados se localizan en los pisos térmicos de la parte superior del premontano, montano bajo y montano.

El bosque nuboso montano tropical es único entre los ecosistemas terrestres ya que está fuertemente vinculado a los ciclos regulares de las formaciones nubosas (Still et al. 1999). Los bosques nubosos se definen como aquellos ecosistemas boscosos cuya ecología y fisiología están vinculadas a su contacto directo con las nubes. Existen bosques nublados en más de 60 países y en algunas islas tropicales y subtropicales. Estos ecosistemas son particularmente frecuentes en los trópicos y subtrópicos de América (Stadtmüller 1987). Los bosques nubosos típicamente albergan una alta proporción de especies endémicas debido a que comúnmente están situados en los picos y crestas de las montañas (Still et al. 1999).

No se conoce exactamente el área que cubren los bosques de niebla, pero se estima que es de 539,263 $\mathrm{km}^{2}$ (Bubb et al. 2002). Sin embargo, los bosques de neblina están desapareciendo rápidamente (Bruijnzeel y Hamilton 2000). Se considera que en Centro y Sur América los bosques nublados abarcan alrededor de 75 millones de hectáreas y 22 millones (30\%) se encuentran protegidas (Kappelle y Brown 2001).
Aparte de que los bosques nublados cubren menor superficie que los bosques latifoliados maduros de tierras bajas y medias, su situación a futuro es extremadamente crítica. Los bosques de niebla tienen una distribución fragmentada en las partes altas de las montañas y están expuestos a mayor fragmentación por la deforestación y la construcción de carreteras. Su principal amenaza la ha constituido la deforestación para dedicar las tierras a la agricultura y la ganadería (Bubb et al. 2002). En el norte de Colombia, más de $90 \%$ de la selva nublada ha desaparecido en pocas décadas debido a su conversión a pastizales (Bruijnzeel y Hamilton 2000).

El aumento de la tecnología de las comunicaciones se ha convertido en otra amenaza para las selvas de neblina ya que sus instalaciones requieren caminos de acceso para construir y mantener las antenas (Bruijnzeel y Hamilton 2000). En la década de 1980 se estimó que la tasa de deforestación de estos ecosistemas era de 20 ha por minuto (Stadtmüller 1987). La deforestación de las tierras altas se estimó en una pérdida anual mundial de $1.1 \%$ lo que es más alto que en cualquier otro ecosistema forestal tropical (Bruijnzeel y Hamilton 2000).

Existen otros problemas en los bosques nublados, tales como el corte y acarreo de leña, la cosecha de productos no maderables, la cacería furtiva, la introducción de especies exóticas y los incendios forestales en aquellos ecosistemas estacionalmente secos. Además, el efecto que el cambio climático global tendrá sobre estos ecosistemas. Se estima que en México, $68 \%$ de los bosques nubosos podrían desaparecer para el 2080 debido al cambio climático global (Ponce-Reyes et al. 2012). Más aun, al unir dos 
Agudelo et al:: Extensión del Bosque Nublado y su Contribución de Lluvia Horizontal a la Precipitación Total

amenazas (deforestación y cambio climático) es posible que $99 \%$ del bosque nuboso de México haya desaparecido para el 2080 (Ponce-Reyes et al. 2012). Se debe tomar en cuenta que los bosques nubosos son el refugio de muchas especies endémicas que se perderán con la pérdida de su hábitat (Still et al. 1999, Bruijnzeel y Hamilton 2000).

Los ecosistemas forestales son esenciales para el control de los procesos erosivos y la hidrología de la cuenca, especialmente en las regiones montañosas con mucha pendiente y precipitación relativamente alta (Cavelier y Goldstein 1989). Los bosques nubosos proveen muchos bienes y servicios ecosistémicos. Desde el punto de vista edáfico, los bosques nublados presentan una capa de residuos orgánicos gruesa y continua, situación que propicia las altas tasas de infiltración. Debido a las bajas temperaturas predominantes, la actividad biológica en los suelos y la meteorización química es reducida. Por lo general, los suelos son ácidos, con pH entre 3.0 y 3.5, a consecuencia de un lavado continuo de nutrimentos.

En términos microclimáticos, los bosques nublados tiene una alta humedad relativa asociada a la precipitación horizontal y a las bajas temperaturas. Esta combinación de factores causa que estos ecosistemas se mantengan permanentemente húmedos. Debido a ello, también existe un alto grado de epifitismo debido a los musgos, líquenes y plantas vasculares de algunas familias como Orchidaceae, Bromeliaceae y Araceae. Además del fuerte epifitismo, estos bosques exhiben un notable endemismo de flora y fauna, propiciado por el efecto biogeográfico de isla (Stadtmüller 1987, Still et al. 1999).

Aparte de todos esos valores, los ecosistemas de montaña son la fuente de todos los grandes ríos del mundo (Price 1998) y los bosques de niebla en particular son importantes para la colecta y regulación del agua. Los sistemas montañosos, con sus bosques nublados incluidos, son los responsables de abastecer el agua dulce para la mitad de la población del mundo (Pratt y Preston 1998). Ante la importancia de los bosques nubosos como depósitos de agua dulce, su gestión cuidadosa, en especial la de los recursos hídricos, debería ser una prioridad absoluta para un mundo que se enfrenta a una crisis de agua sin precedentes (Liniger y Weingartner 1998).

Por precipitación se entiende todas las aguas meteóricas que caen sobre la superficie terrestre en forma líquida o sólida. La precipitación que se deposita por la gravedad, ya sea en forma de lluvia, llovizna, chubasco, nieve o granizo, se conoce como lluvia vertical o normal. Además, existe la precipitación horizontal u oculta, lluvia del bosque o intercepción horizontal.

La precipitación horizontal es un proceso mediante el cual las gotas pequeñas de vapor de agua en movimiento horizontal entran en contacto con la vegetación. A diferencia de la precipitación vertical que no está influenciada por la vegetación en términos de su monto y distribución, la precipitación horizontal está fuertemente relacionada con la presencia de los bosques nublados en estado maduro (Stadtmüller 1987, Bruijnzeel y Hamilton 2000).

En las cuencas hidrográficas de montaña de América tropical y subtropical, las tierras cubiertas con bosques nublados aportan considerables volúmenes adicionales de agua. Tales aportes varían entre 7 y $158 \%$ sobre la precipitación vertical o normal (Stadtmüller 1987). Ante esta importancia hidrológica de los bosques nublados, como cinturones de condensación del vapor de agua atmosférica, se ha investigado la cuantificación de la precipitación horizontal. Estas investigaciones, desde principio de 1900, han estado concentradas en las regiones templadas de Europa, Estados Unidos y Japón. En las regiones tropical y subtropical, las investigaciones sobre la precipitación horizontal empezaron desde hace unos 50 años debido a que la importancia hidrológica de estos bosques es de un reconocimiento más reciente (Cáceres 1981). En Centro América son pocos los estudios para cuantificar el aporte adicional de agua de los bosques nublados a las cuencas hidrográficas. Quizá los más destacados esfuerzos orientados a la cuantificación de la precipitación horizontal son los sistemas montañosos de Las Minas en Guatemala, La Selva y Balalaika en Costa Rica, El Merendón en Honduras y El Trifinio en El Salvador (Stadtmüller y Agudelo 1990, Ramírez 2011).

De las 237.1 ha de la zona núcleo de la Reserva Biológica Uyuca, solamente 52.0 ha están cubiertas con bosque nublado latifoliado maduro (Figura 1). El impacto hidrológico de este bosque para la Escuela Agrícola Panamericana (Zamorano) en producción de agua es invaluable así como para las comunidades del pie de monte del cerro Uyuca. Esta porción de la montaña, que es recolectora de agua, aunada a la precipitación vertical en ella, son las responsables de garantizar el suministro continuo y sostenido de este líquido para todos los usuarios de la Escuela Agrícola 
Panamericana y más de 10 comunidades.

. El conocimiento del aporte de la precipitación horizontal por parte de este bosque nublado es un importante instrumento de manejo de los recursos a nivel institucional. Ante esta importancia se procedió con un estudio en este sentido con los objetivos específicos de determinar la cantidad de lluvia en el bosque latifoliado del cerro Uyuca, delimitar en forma precisa la superficie cubierta por este tipo de bosque en estado maduro, determinar la precipitación horizontal dentro de la masa forestal, comparar las precipitaciones en campo abierto y bajo del dosel del bosque y evaluar el límite actual de la zona impactada por nubes o neblinas en la Reserva Biológica Uyuca.
El estudio de la precipitación fue con registros diarios, la nubosidad y la visibilidad, la dirección y la velocidad del viento entre 1987 y 1992. La precipitación a campo abierto la obtuvimos de tres pluviómetros estándar instalados en un área aledaña al bosque maduro. Registramos la precipitación dentro del bosque con 12 pluviómetros construidos y distribuidos en varias altitudes dentro del bosque latifoliado (Cuadro 1) con la colaboración del bioclimatólogo alemán Thomas Stadtmüller. Los pluviómetros eran embudos con $52 \mathrm{~cm}$ de diámetro. Unimos estos aparatos con mangueras a recipientes plásticos con capacidad de $18.9 \mathrm{~L}$ (5 galones).

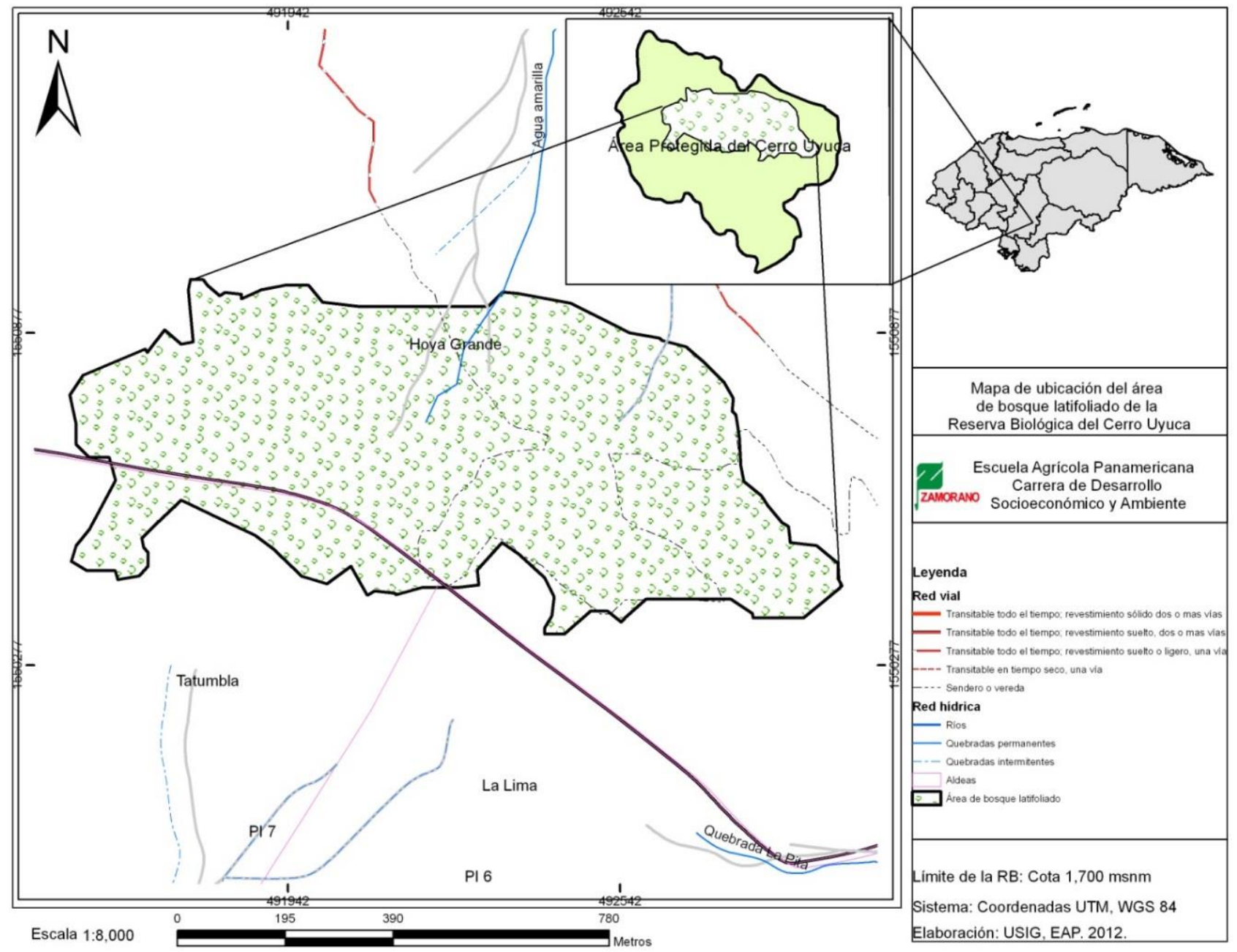

Figura 1. Ubicación del bosque latifoliado maduro (zona de estudio de 52 ha) de la zona núcleo de la Reserva Biológica Uyuca, Honduras. 
Cuadro 1. Ubicación geográfica (coordenadas) de 15 pluviómetros (del 1 al 3 en área abierta y 12 dentro del bosque latifoliado maduro) en la Reserva Biológica Uyuca, Honduras.

\begin{tabular}{cccc}
\hline & & \multicolumn{2}{c}{ Coordenadas } \\
\cline { 3 - 4 } Pluviómetro & $\begin{array}{c}\text { Altura } \\
\text { (msnm) }\end{array}$ & $\mathbf{X}$ & $\mathbf{Y}$ \\
\hline 1 & 1,876 & 492510 & 1550696 \\
2 & 1,873 & 492457 & 1550652 \\
3 & 1,895 & 492556 & 1550583 \\
A1 & 1,839 & 492301 & 1550709 \\
A2 & 1,789 & 492224 & 1550827 \\
A3 & 1,891 & 492290 & 1550761 \\
A4 & 1,898 & 492195 & 1550657 \\
B1 & 1,910 & 492169 & 1550524 \\
B3 & 1,908 & 492250 & 1550608 \\
B2 & 1,909 & 492185 & 1550516 \\
B4 & 1,916 & 492696 & 1550462 \\
C1 & 1,975 & 492180 & 1550439 \\
C2 & 1,969 & 492365 & 1550488 \\
C3 & 1,916 & 492511 & 1550381 \\
C4 & 1,991 & 492530 & 1550398 \\
\hline
\end{tabular}

En el 2011 levantamos un mapa planimétrico del bosque latifoliado maduro de la RBU, georeferenciamos las redes de pluviómetros instaladas dentro y fuera del bosque y redefinimos los límites de la zona impactada por las nubes o las neblinas. Para evaluar el grado de epifitismo (Waechter 1998), necesario para la redefinición mencionada, utilizamos la siguiente escala:

Epifitismo bajo. Pocos individuos de Bromeliaceae, Araceae, Orquidaceae, musgos y helechos en troncos, ramas y copas de los árboles. Los sitios con este grado de epifitismo tienen por lo general una humedad relativa baja y la precipitación anual de baja a moderada.

Epifitismo medio. Presencia moderada de individuos pertenecientes a los grupos vegetales mencionados. La humedad relativa y la precipitación se incrementan de manera considerable. En estos sitios, la precipitación anual puede ser de 1,600 a 1,800 mm.
Epifitismo alto. Fuerte presencia de individuos de todos los grupos vegetales mencionados. En algunas localidades los troncos y copas de los árboles están casi completamente cubiertos con epífitas. En estos sitios la humedad relativa es alta, generalmente mayor a $80 \%$, y la precipitación anual es de $1,900 \mathrm{~mm}$ o más sin períodos largos de sequía.

Empleamos la escala propuesta por Stadtmüller (1987, Cuadro 2) para determinar la visibilidad o nubosidad. Además, aplicamos la escala de Beaufort (Singleton 2008) para estimar la velocidad del viento. Esta es una medida empírica de dicha variable y está basada principalmente en el estado de las olas del mar y la fuerza del viento. También se puede usar por su efecto sobre objetos terrestres (Cuadro 3). Para evaluar la dirección del viento empleamos una brújula Suunto.

Cuadro 2. Características del grado de nubosidad y visibilidad de un sitio dado según Stadtmüller (1987).

\begin{tabular}{|c|c|c|}
\hline Grado & $\begin{array}{l}\text { Término } \\
\text { descriptivo }\end{array}$ & Características \\
\hline 0 & Claro & $\begin{array}{l}\text { No existe en el sitio o } \\
\text { bosque problema de } \\
\text { visibilidad. Hay muy pocas } \\
\text { nubes o neblinas en el } \\
\text { ambiente. }\end{array}$ \\
\hline 1 & $\begin{array}{l}\text { Ligeramente } \\
\text { nublado }\end{array}$ & $\begin{array}{l}\text { Los sitios con incidencia } \\
\text { de las nubes o las } \\
\text { neblinas, pero pueden } \\
\text { observarse los objetos a } \\
\text { más de } 100 \mathrm{~m} \text { de } \\
\text { distancia. }\end{array}$ \\
\hline 2 & Nublado & $\begin{array}{l}\text { Los sitios con incidencia } \\
\text { de las nubes o las } \\
\text { neblinas densas. Los } \\
\text { objetos no se pueden } \\
\text { observar a más de } 100 \mathrm{~m} \\
\text { de distancia. }\end{array}$ \\
\hline 3 & $\begin{array}{l}\text { Muy } \\
\text { nublado }\end{array}$ & $\begin{array}{l}\text { Los sitios en donde la } \\
\text { densidad de las nubes o } \\
\text { las neblinas es tal que no } \\
\text { es posible diferenciar los } \\
\text { objetos a una distancia de } \\
10 \mathrm{~m} \text {. }\end{array}$ \\
\hline
\end{tabular}


Para la metodología de evaluación estimamos la cantidad de la precipitación vertical y horizontal bajo el concepto de año hidrológico. En Honduras, el año hidrológico se inicia el primero de mayo y finaliza el 30 de abril del siguiente año. De todos los registros diarios de todos los elementos climáticos involucrados en el estudio para el período 1987-92, calculamos los siguientes valores.

Precipitación mensual, obtenida así:

$\mathrm{Pm}=\sum \mathrm{Pd}$,

Donde:

$\mathrm{Pm}=$ precipitación mensual y

$\mathrm{Pd}=$ precipitación de cada día .

Precipitación total anual, calculada mediante: $\mathrm{Pta}=\sum \mathrm{Pah}$,
Donde:

$\mathrm{Pta}=$ precipitación total anual y

Pah = precipitación de cada año hidrológico.

Precipitación promedio mensual, determinada así: $\mathrm{Ppm}=\Sigma \mathrm{Pm} / \mathrm{n}$,

Donde:

Ppm = precipitación mensual de cada año,

$\mathrm{Pm}=$ precipitación mensual y

$\mathrm{n}=$ número de años de registro.

Precipitación promedio total anual, obtenida así: Ppta $=\sum$ Pta/n

Donde:

Ppta $=$ precipitación promedio total anual,

$\mathrm{Pta}=$ precipitación total anual y

$\mathrm{n}=$ número de años registro.

Cuadro 3. Descripción de la velocidad del viento según la escala de Beaufort (Singleton 2008).

\begin{tabular}{cllc}
\hline $\begin{array}{c}\text { Número } \\
\text { Beaufort }\end{array}$ & Término descriptivo & Efectos sobre objetos terrestres & $\begin{array}{c}\text { Velocidad } \\
\text { m/s }\end{array}$ \\
\hline 0 & Calma & El humo asciende verticalmente & $0.0-0.2$ \\
\hline 1 & Aire ligero & $\begin{array}{l}\text { Se manifiesta por la desviación del muro, no de la } \\
\text { veleta }\end{array}$ & $0.3-1.5$ \\
\hline 2 & Brisa ligera & $\begin{array}{l}\text { El viento se siente en la cara; las hojas susurran; se } \\
\text { mueve la veleta }\end{array}$ & $1.6-3.3$ \\
\hline 3 & Brisa suave & Se mueven las hojas y las ramillas pequeñas & $3.4-5.4$ \\
\hline 4 & Brisa moderada & Se levanta el polvo; se mueven las ramas pequeñas & $5.5-7.9$ \\
\hline 5 & Brisa fuerte & Se mueven los árboles pequeños & $8.0-10.7$ \\
\hline 6 & Viento fuerte & $\begin{array}{l}\text { Se mueven las ramas grandes; los paraguas se } \\
\text { manejan con dificultad }\end{array}$ & $10.8-13.8$ \\
\hline 7 & Viento muy fuerte & $\begin{array}{l}\text { Se mueven los árboles grandes; hay dificultad de } \\
\text { caminar contra el viento }\end{array}$ & $13.9-17.1$ \\
\hline 8 & Viento borrascoso & Se quiebran las ramas & $17.2-20.7$ \\
\hline 9 & Tormenta de viento & Se producen daños ligeros en las casas & $20.8-24.4$ \\
\hline 10 & Tormenta fuerte & $\begin{array}{l}\text { Se producen daños considerables; se desraízan los } \\
\text { árboles }\end{array}$ & $24.5-28.5$ \\
\hline
\end{tabular}

Comparamos los datos de precipitación con el grado de nubosidad 0 visibilidad. Además, determinamos las posibles variaciones que ocurrieron en este periodo de tiempo refiriéndose a los factores tales como la dirección y la velocidad del viento.

El área abierta era relativamente plana y la geomorfología del terreno tuvo poco impacto sobre la precipitación vertical. Por tal razón, se calculó la precipitación de esta zona con un promedio aritmético simple. El área del bosque maduro incluye formas de terreno variables: terrenos algo planos, crestas, áreas cóncavas y áreas convexas. Esta variabilidad topográfica tuvo un marcado efecto en la cantidad de precipitación que cayó dentro de cada pluviómetro. 
Ante esta situación, la precipitación promedio dentro del bosque se estimó con el método de los polígonos de Thiessen (Chow et al. 1994). Los datos crudos de cada pluviómetro (no incluidos) denotan la irregularidad, no solo mensual, sino debida al área determinada para cada polígono.

El método de los polígonos de Thiessen se aplica cuando las medidas de precipitación en los pluviómetros sufren variaciones (Chow et al. 1994). Este método fue necesario porque los 12 pluviómetros diseñados y construidos para este estudio, tenían una apertura mayor de $20 \mathrm{~cm}$ del pluviómetro estándar.

Para cada pluviómetro determinamos su área de influencia y luego obtuvimos un factor de ponderación. El procedimiento consistió en unir los pluviómetros adyacentes con líneas rectas para formar triángulos. Luego se trazaron mediatrices a las líneas que unen los pluviómetros hasta su encuentro dentro del triángulo (una mediatriz es una línea recta perpendicular a un segmento de recta y que parte de su punto medio). Las mediatrices se prolongan hasta el límite de la cuenca o unidad a estimar, en este caso, el bosque latifoliado maduro. Luego se calculó el área formada por las mediatrices para cada pluviómetro. Así, cada pluviómetro cubrió un sector del bosque o polígono particular (Figura 2). Calculamos el ponderador del área como el cociente entre el área de cada pluviómetro y el área total (por esto su suma es 1). La precipitación ponderada la obtuvimos al multiplicar la precipitación medida en cada pluviómetro y el factor ponderador del área (Chow et al. 1994).

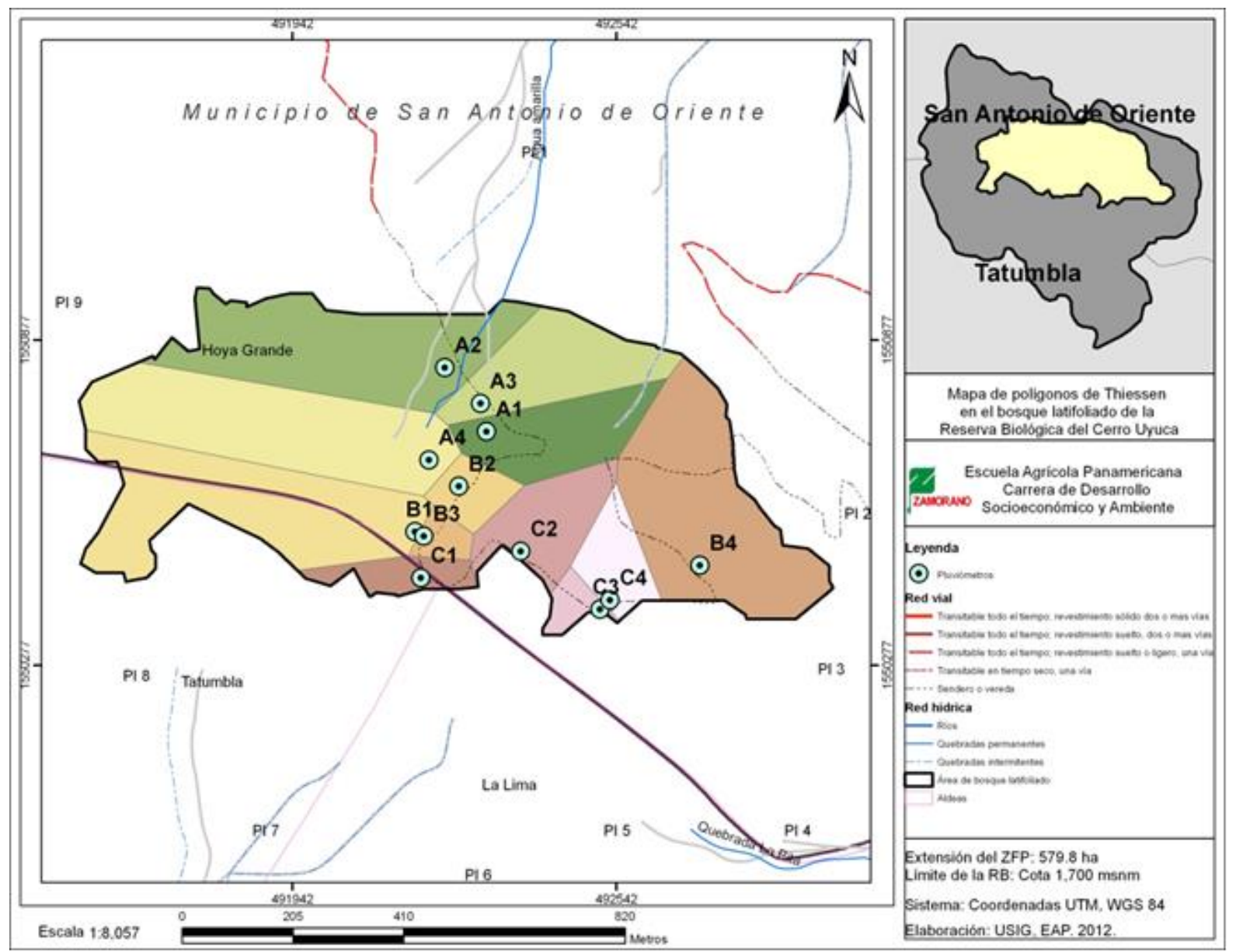

Figura 2. Ubicación de los polígonos de Thiessen con su área ponderada específica en el bosque latifoliado maduro del área núcleo de la Reserva Biológica Uyuca, Honduras. 
La precipitación media es:

$\mathrm{P}=\Sigma(\mathrm{Pi} \times \mathrm{Ai}) / \mathrm{A}=\Sigma((\mathrm{Pi} \times \mathrm{Ai} / \mathrm{A})$.

$\sum$ va de i a n.

Donde:

$\mathrm{P}=$ precipitación media sobre la cuenca

$\mathrm{Pi}=$ precipitación observada en la Estación $\mathrm{i}$

$\mathrm{Ai}=$ área del polígono correspondiente a la Estación $\mathrm{i}$

$\mathrm{A}=$ área total de la cuenca

$\mathrm{n}=$ número de pluviómetros con influencia en la

cuenca o área de estudio.

La ponderación se determina como:

$\mathrm{D}=\Sigma \mathrm{i}$ ai $\mathrm{Di} / \mathrm{A}$ (Aparicio 2006).

Donde:

$\mathrm{D}=$ altura de precipitación media $(\mathrm{mm})$.

ai = área de influencia de la estación $\left(\mathrm{km}^{2}\right)$.

$\mathrm{Di}=$ precipitación media en la estación $\mathrm{i}(\mathrm{mm})$.

$\mathrm{A}=$ área total de la cuenca $\left(\mathrm{km}^{2}\right)$.

La precipitación para cada año la obtuvimos al sumar los valores parciales para cada área de influencia. Extrapolamos los valores de la precipitación de los polígonos a toda el área del bosque maduro. Finalmente, calculamos la precipitación promedio para el bosque maduro por medio de la sumatoria de los valores anuales dividido entre el número de los años de registro.

\section{Resultados y Discusión}

La precipitación promedio total anual varió desde $972 \mathrm{~mm}$ en el pluviómetro A4 a $4,152 \mathrm{~mm}$ en el pluviómetro C4 (Figura 2). No analizamos los datos para determinar si hubo algún cambio climático en los registros diarios de precipitación dentro del bosque latifoliado maduro. Sin embargo, el 31 de mayo de 1988 en el pluviómetro C4 se registró una precipitación de $293.6 \mathrm{~mm}$ en un período de 24 horas. El promedio anual mínimo para los 12 pluviómetros fue de $747 \mathrm{~mm}$ y el máximo de $3,316 \mathrm{~mm}$ (Cuadro 4). El promedio mensual mínimo para los cinco años de estudio fue de $62.3 \mathrm{~mm}$ en marzo y el promedio mensual máximo fue de $276.3 \mathrm{~mm}$ en septiembre (Cuadro 4).

Un mes húmedo tiene aproximadamente $100 \mathrm{~mm}$ de precipitación (Wadsworth 2000). El período comprendido entre mayo y febrero tuvo una precipitación de $1,892 \mathrm{~mm}$ y en los meses relativamente secos de marzo y abril se registraron $129 \mathrm{~mm}$ de lluvia (Figura 3). Esto significa que dentro del bosque, $94 \%$ de la precipitación ocurrió en 10 meses, con una relativa época de sequía a nivel de atmósfera, que se extendió por dos meses (Cuadro 4). Como resultado, el suelo del bosque maduro de Uyuca se mantiene permanentemente húmedo y el exceso de precipitación se traslada por procesos de infiltración hacia las partes bajas de la montaña y a los mantos freáticos a nivel del valle.

Cuadro 4. Precipitación total y promedio con desviación estándar registrada en 12 pluviómetros en cada mes durante cinco años hidrológicos (de mayo 1987 a abril 1992) dentro del bosque nublado de la Reserva Biológica Uyuca, Honduras.

\begin{tabular}{lrcc}
\hline & \multicolumn{3}{c}{ Precipitación (mm) } \\
\cline { 2 - 4 } Mes & Total & Promedio & $\begin{array}{c}\text { Desviación } \\
\text { Estándar }\end{array}$ \\
\hline Mayo & 1,964 & 163.7 & 6.9 \\
Junio & 3,100 & 258.3 & 93.5 \\
Julio & 2,885 & 240.4 & 119.1 \\
Agosto & 3,052 & 254.3 & 106.5 \\
Septiembre & 3,316 & 276.3 & 102.9 \\
Octubre & 1,787 & 148.9 & 42.6 \\
Noviembre & 1,691 & 140.9 & 63.7 \\
Diciembre & 1,731 & 144.3 & 85.2 \\
Enero & 2,027 & 168.9 & 95.3 \\
Febrero & 1,152 & 96.0 & 54.2 \\
Marzo & 747 & 62.3 & 29.9 \\
Abril & 807 & 67.3 & 19.5 \\
\hline \multicolumn{3}{l}{ Total anual del promedio mensual $2,021.6$} \\
\hline \multicolumn{4}{c}{}
\end{tabular}

La alta precipitación dentro del bosque maduro obedece, en términos climáticos, a la elevación de la cordillera, al impacto de nubes o neblinas sobre la montaña y a la velocidad y dirección del viento. Con excepción de mayo, se registraron vientos entre 1.6 y $5.4 \mathrm{~m} / \mathrm{s}$, lo que indica que el viento sopló con brisa ligera a brisa suave (Cuadro 4). Estas condiciones del viento en el bosque maduro fueron determinantes para inducir elevadas precipitaciones. En todos los meses del año, los vientos dominantes fueron del NE con algunas variantes hacia el NW (Cuadro 5). Tal situación confirma que los vientos que dominan los regímenes pluviométricos en el valle de Zamorano y en el cerro Uyuca son del NE. Tales vientos son los que manejan los regímenes de precipitación vertical a escala regional de Centroamérica (Vargas 2006). 
Seis meses del año tuvieron tramos con pocas nubes o neblinas en el ambiente (Cuadro 5). En parte de estos meses, así como en todos los restantes, hubo una nubosidad o visibilidad entre ligeramente nublado a muy nublado. La relación entre la elevación, la velocidad y la dirección del viento, la nubosidad y la presencia del bosque maduro multiestratificado fueron factores decisivos para propiciar las altas tasas de precipitación que existen en este ecosistema.

Entre 1989 y 1990 se registró el valor mínimo de precipitación promedio anual $(2,205 \mathrm{~mm})$ y el valor máximo (5,229 mm) en 1991-1992 (Cuadro 6). En el período 1987-1990 hubo poca variación en la precipitación anual ponderada, con un mínimo de $2,205 \mathrm{~mm}$ y un máximo de 2,306 $\mathrm{mm}$. Se desconoce si el período 1987-1990 correspondió a un ciclo hidrológico seco y si la precipitación anual entre 1991 y 1992 obedeció a un ciclo húmedo o a situaciones atmosféricas especiales tales como huracanes 0 tormentas tropicales.

La precipitación promedio total anual para el bosque maduro fue de 2,360 $\mathrm{mm}$ (Cuadro 6). Esta precipitación difiere en $338 \mathrm{~mm}$ del valor de 2,022 mm correspondiente al registro pluviométrico. Tal diferencia radica esencialmente en la técnica utilizada para la estimación de los datos. Con el método de los polígonos de Thiessen el valor promedio anual en el bosque maduro de la RBU fue de 2,360.2 mm (Cuadro 7).

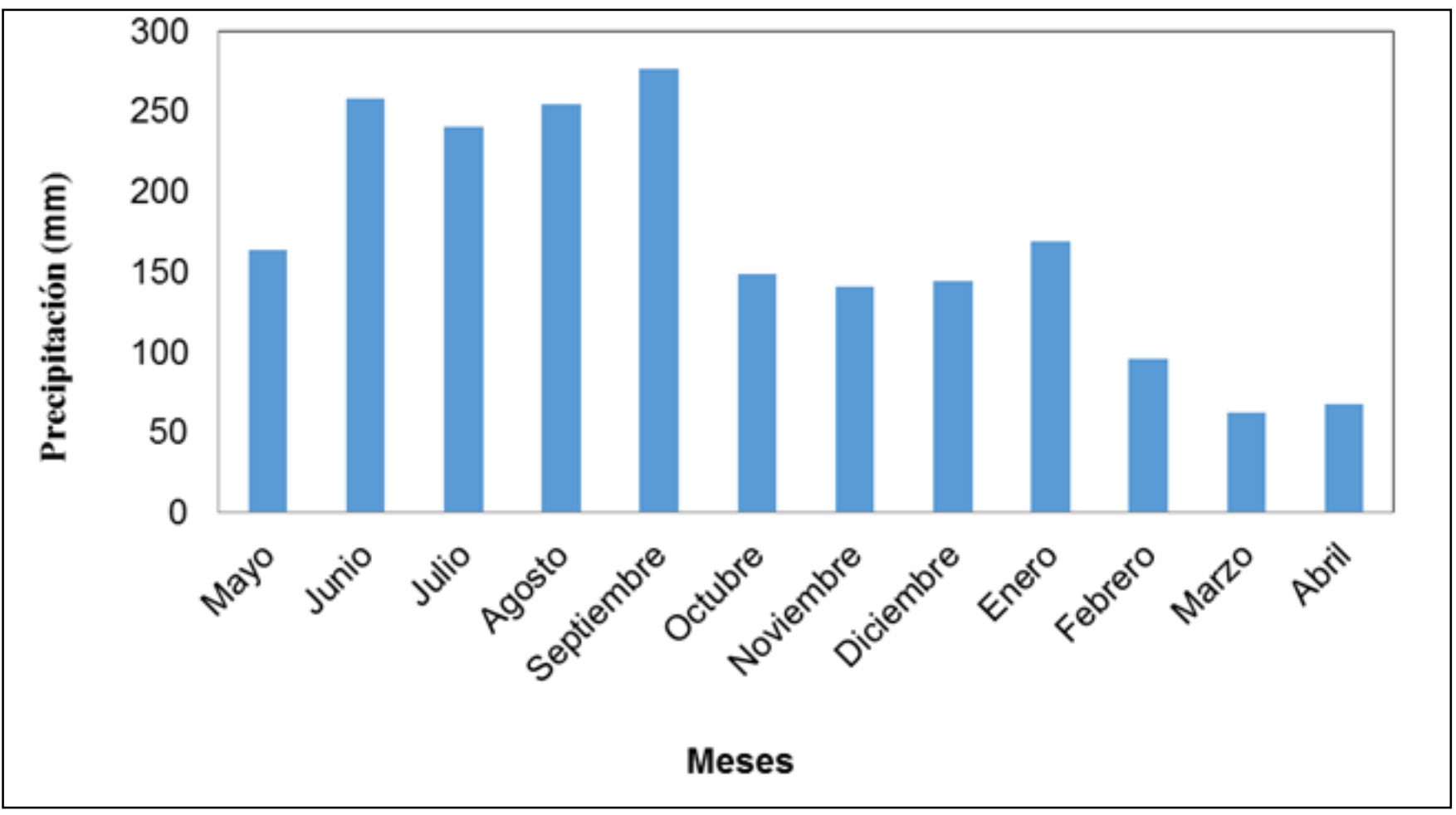

Figura 3. Precipitación promedio mensual para cinco años hidrológicos de registro (1987-1992), según las medidas tomadas en 12 pluviómetros instalados dentro del bosque latifoliado de la Reserva Biológica Uyuca, Honduras. 
Cuadro 5. Valor promedio por mes de la velocidad y la dirección del viento, de la nubosidad y de la visibilidad para el período de registro 1987-1992, en el bosque maduro de la Reserva Biológica Uyuca, Honduras.

\begin{tabular}{lccl}
\hline Mes & Velocidad del viento $(\mathbf{m} / \mathbf{s})$ & Dirección del viento & \multicolumn{1}{c}{ Nubosidad y visibilidad } \\
\hline Mayo & $3.4-5.4$ & NE- NW & Claro y ligeramente nublado \\
Junio & $1.6-5.4$ & NE- NW & Ligeramente nublado y muy nublado \\
Julio & $1.6-5.4$ & NE- NW & Ligeramente nublado y nublado \\
Agosto & $1.6-5.4$ & NE & Ligeramente nublado \\
Septiembre & $1.6-5.4$ & NE & Ligeramente nublado \\
Octubre & $1.6-5.4$ & NE- NW & Claro y ligeramente nublado \\
Noviembre & $1.6-5.4$ & NE & Claro y ligeramente nublado \\
Diciembre & $1.6-5.4$ & NE- NW- SE & Ligeramente nublado y nublado \\
Enero & $1.6-5.4$ & NE-W & Ligeramente nublado \\
Febrero & $1.6-5.4$ & NE & Claro y ligeramente nublado \\
Marzo & $1.6-5.4$ & NE-NW & Claro ligeramente nublado y nublado \\
Abril & $1.6-5.4$ & NE-NW & Claro y ligeramente nublado \\
\hline
\end{tabular}

Cuadro 6. Valor total anual ponderado para los años hidrológicos de los registros de la precipitación promedio total anual (en $\mathrm{mm}$ ) estimados mediante el método de los polígonos de Thiessen para el bosque maduro de la Reserva Biológica Uyuca, Honduras.

\begin{tabular}{lcc}
\hline $\begin{array}{c}\text { Años de } \\
\text { registros }\end{array}$ & $\begin{array}{c}\text { Precipitación } \\
\text { anual } \\
\text { ponderada } \\
(\mathbf{m m})\end{array}$ & $\begin{array}{c}\text { Precipitación } \\
\text { anual promedio } \\
(\mathbf{m m})\end{array}$ \\
\hline $1987-1988$ & 2,306 & 2,085 \\
$1988-1989$ & 2,260 & 2,022 \\
$1989-1990$ & 2,205 & 1,951 \\
$1990-1991$ & 2,271 & 2,051 \\
$1991-1992$ & 5,229 & 3,693 \\
\hline Promedio total anual & 2,854 & 2,360 \\
\hline
\end{tabular}

La precipitación promedio mensual para el periodo 1987-92 registrada en los pluviómetros instalados a campo abierto tuvo un valor mínimo de $745 \mathrm{~mm}$ en el pluviómetro 3 y un valor máximo de $1,393 \mathrm{~mm}$ en el pluviómetro 1 (Cuadro 8). En los pluviómetros 2 y 3 registramos precipitaciones similares, mientras que en el pluviómetro 1 tuvimos una precipitación casi el doble de la de los otros dos pluviómetros. Esta marcada diferencia en precipitación podría deberse a la mayor proximidad del pluviómetro 1 al bosque maduro.
Cuadro 7. Precipitación promedio anual $(\mathrm{mm})$ de cinco años hidrológicos (de 1987 a 1992) con su desviación estándar (DE), calculados mediante el método de polígonos de Thiessen para cada pluviómetro, para el bosque maduro del cerro Uyuca, Honduras.

\begin{tabular}{ccccr}
\hline $\begin{array}{l}\text { Pluvió- } \\
\text { metro }\end{array}$ & $\begin{array}{c}\text { Área } \\
\text { (ha) }\end{array}$ & $\begin{array}{c}\text { Ponde- } \\
\text { rador } \\
\text { de área }\end{array}$ & $\begin{array}{c}\text { Precipita- } \\
\text { ción } \\
\text { promedio } \\
\text { anual } \\
\text { (mm) }\end{array}$ & \multicolumn{1}{c}{ DE } \\
\hline A1 & 4.08 & 0.12 & $1,517.6$ & 30.9 \\
A2 & 3.76 & 0.11 & $1,590.6$ & 130.6 \\
A3 & 3.99 & 0.12 & $2,025.4$ & 137.7 \\
A4 & 1.71 & 0.05 & 973.2 & 190.4 \\
B1 & 0.84 & 0.03 & $1,598.2$ & 200.5 \\
B2 & 2.16 & 0.07 & $2,714.4$ & 185.4 \\
B3 & 0.95 & 0.03 & $1,608.0$ & 85.0 \\
B4 & 1.21 & 0.04 & $1,566.2$ & 150.2 \\
C1 & 6.76 & 0.21 & $5,250.0$ & $4,794.9$ \\
C2 & 1.59 & 0.05 & $1,732.6$ & 117.3 \\
C3 & 1.89 & 0.06 & $3,595.0$ & $3,220.5$ \\
C4 & 3.87 & 0.12 & $4,151.6$ & 310.7 \\
\hline Promedio & & $2,360.2$ & \\
\hline
\end{tabular}

Área = área de influencia del pluviómetro

Ponderador de área $=\mathrm{El}$ ponderador de área se calcula como el cociente entre el área asociada a cada pluviómetro y el área total (por eso su suma es 1 ). 
Cuadro 8. Precipitación mensual por pluviómetro y total mensual y anual $(\mathrm{mm})$ para tres pluviómetros instalados a campo abierto en la Reserva Biológica Uyuca, Honduras.

\begin{tabular}{lrrrr}
\hline & \multicolumn{3}{c}{ Pluviómetros } & \\
\cline { 2 - 4 } Mes & $\mathbf{1}$ & $\mathbf{2}$ & $\mathbf{3}$ & Total \\
\hline Mayo & 162 & 154 & 154 & 470 \\
Junio & 223 & 141 & 140 & 504 \\
Julio & 142 & 64 & 64 & 270 \\
Agosto & 198 & 89 & 89 & 376 \\
Septiembre & 272 & 127 & 89 & 488 \\
Octubre & 118 & 60 & 60 & 238 \\
Noviembre & 84 & 44 & 44 & 172 \\
Diciembre & 46 & 30 & 30 & 107 \\
Enero & 47 & 27 & 27 & 100 \\
Febrero & 28 & 15 & 15 & 58 \\
Marzo & 21 & 11 & 11 & 43 \\
Abril & 52 & 34 & 22 & 108 \\
\hline Total & 1,393 & 796 & 745 & 2,934 \\
\hline
\end{tabular}

La precipitación promedio mensual mínima para los tres pluvímetros ocurrió en febrero (58 mm) mientras que la máxima $(488 \mathrm{~mm})$ fue en septiembre (Cuadro 8). En el período comprendido entre mayo y octubre cayó el $80 \%$ de la precipitación anual. En el transcurso de la temporada lluviosa, en julio, se registraron solamente $270 \mathrm{~mm}$ de precipitación debido posiblemente al efecto de la canícula en Centroamérica (Vargas 2006).

En el área abierta, la época de sequía abarca aproximadamente seis meses, de noviembre a abril, y febrero y marzo son los meses más secos con 101 $\mathrm{mm}$ de precipitación (Cuadro 8), equivalentes a $3.4 \%$ del total anual. La precipitación promedio total anual en el área abierta, según el promedio aritmético para los tres pluviómetros, fue de $978 \mathrm{~mm}$. Este valor tiene una diferencia de $68 \mathrm{~mm}$ de lluvia anual con respecto al promedio total anual de $1,046 \mathrm{~mm}$ de la estación Zamorano con 60 años de registro (Cuadro 9).

Determinamos la precipitación horizontal por medio de la diferencia entre la precipitación que cayó dentro del bosque y la que se depositó a campo abierto. Debido a que el método de los polígonos de Thiessen arroja información más precisa sobre la precipitación promedio sobre un área topográfica accidentada, tomamos como referencia este valor. La precipitación promedio total anual dentro del bosque fue de $2,360.2 \mathrm{~mm}$ y a campo abierto fue $978 \mathrm{~mm}$. Por tanto, la precipitación horizontal fue de $1,382.2 \mathrm{~mm}$. Basado en este dato, se deduce que el bosque latifoliado maduro del Cerro Uyuca aportó 141.3\% adicional de lluvia. Este significativo aporte del bosque maduro nublado de Uyuca constituye una garantía para la producción continua y sostenible de agua para los sitios más bajos y secos de la montaña. GarcíaSantos et al. (2004) señalaron que hay que tomar en cuenta que al separar y medir la precipitación horizontal de la precipitación total es impreciso por lo que provee solo un estimado inicial de la precipitación horizontal neta que capta el suelo. Consecuentemente, la lluvia horizontal bien podría haber contribuido el doble de la precipitación normal caída durante los cinco meses de sequía de su sitio de trabajo en España.

Cuadro 9. Valor medio mensual y anual de la temperatura y la biotemperatura para 28 años de registro (1973-2001), y precipitación media mensual y promedio anual para 60 años de registro (1944-2004) en Zamorano, Honduras (Fuente: Estación Zamorano).

\begin{tabular}{lccc}
\hline & \multicolumn{3}{c}{ Elementos climáticos } \\
\cline { 2 - 4 } Mes & $\begin{array}{c}\text { Tempe- } \\
\text { ratura } \\
\left({ }^{\circ} \mathbf{C}\right)\end{array}$ & $\begin{array}{c}\text { Biotem- } \\
\text { peratura } \\
\left({ }^{\circ} \mathbf{C}\right)\end{array}$ & $\begin{array}{c}\text { Precipita- } \\
\text { ción } \\
(\mathbf{m m})\end{array}$ \\
\hline Enero & 22.5 & 22.5 & 11 \\
Febrero & 23.4 & 23.4 & 8 \\
Marzo & 25.2 & 24.6 & 10 \\
Abril & 27.0 & 23.2 & 39 \\
Mayo & 26.4 & 24.0 & 141 \\
Junio & 25.1 & 24.6 & 181 \\
Julio & 24.4 & 24.3 & 134 \\
Agosto & 24.7 & 24.5 & 150 \\
Septiembre & 24.6 & 24.3 & 184 \\
Octubre & 24.2 & 24.2 & 126 \\
Noviembre & 23.2 & 23.4 & 47 \\
Diciembre & 22.6 & 22.6 & 14 \\
\hline Promedio & 24.4 & 23.8 & 1,046 \\
\hline
\end{tabular}

Independientemente del método utilizado para calcular la precipitación promedio total anual dentro del bosque maduro, este valor fue superior a los 2,000 $\mathrm{mm}(2,022 \mathrm{~mm}$ según promedio aritmético) e inferior a 
los $3,000 \mathrm{~mm}(2,854 \mathrm{~mm}$, según el método de los polígonos de Thiessen ponderado, cuadro 6). Con esta información se deduce que el bosque maduro corresponde en su totalidad a la zona de vida bosque muy húmedo montano bajo subtropical. En general, este ecosistema tiene un ámbito de precipitación promedio total anual de entre 2,000 y $4,000 \mathrm{~mm}$. La fisonomía de la comunidad vegetal madura de esta zona de vida confirma la información climática derivada de este estudio.

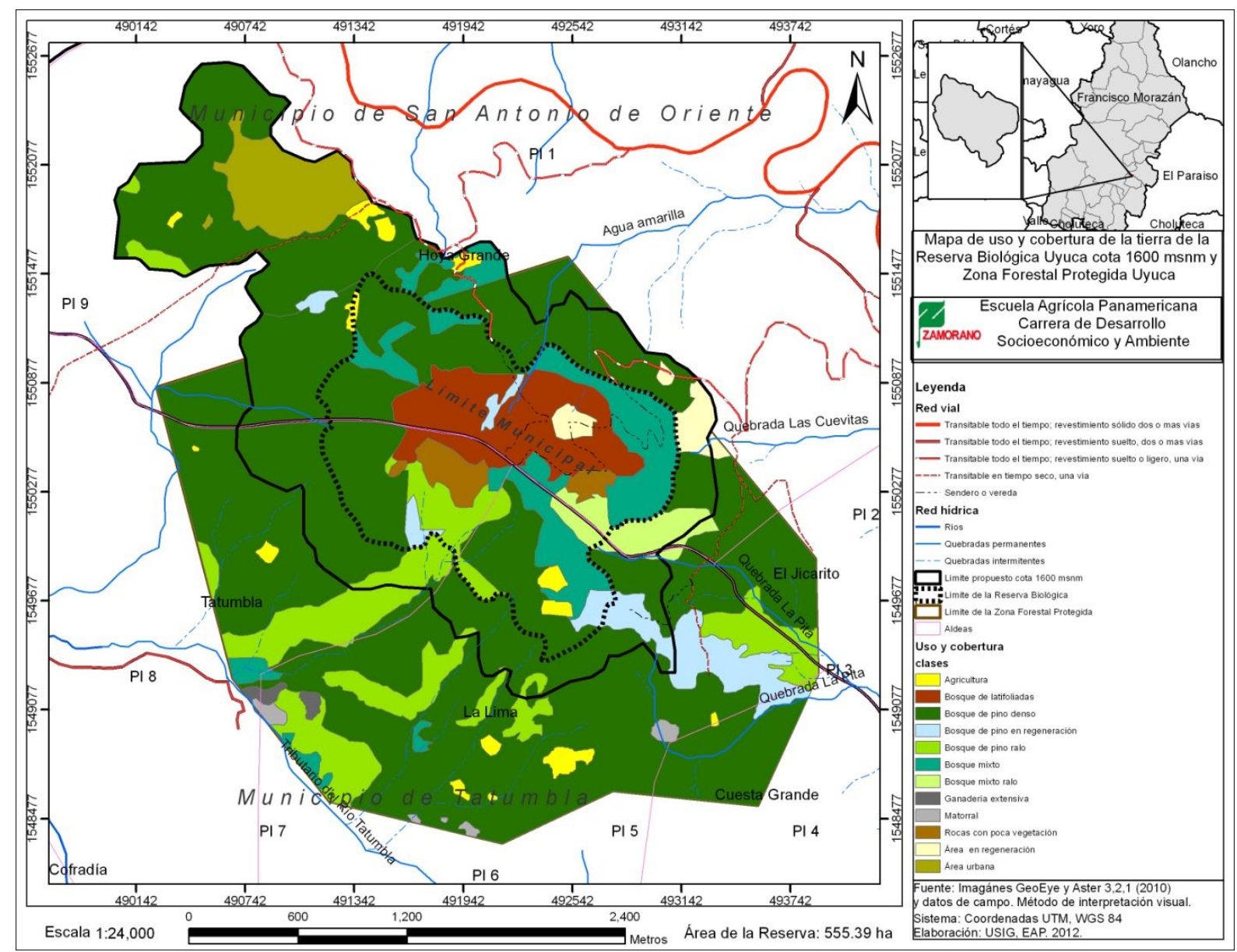

Figura 4. Límite de la zona impactada por las nubes o las neblinas, cota 1,600 msnm, en relación a la zona núcleo de la reserva biológica, cota 1,700 msnm y la zona de amortiguamiento (zona forestal protegida según el Decreto Ejecutivo Número 211 - 85 de 1985) en el Cerro Uyuca, Honduras.

Zamorano ha garantizado por más de 60 años la protección del cerro Uyuca contra los incendios forestales, los cortes ilegales de madera, la extracción de los productos del bosque nublado y la cacería furtiva. Desde 1981, Zamorano también ha propiciado la restauración en forma natural de los sitios que estuvieron bajo la actividad agrícola. Como resultado de este proceso de protección y restauración, la parte superior del cerro Uyuca tiene una cobertura de bosque natural en varias etapas de sucesión, los árboles tienen bastante epifitismo en los troncos, las ramas y las copas. En las partes más bajas hay rodales puros y mixtos de pinabete (Pinus maximinol) y en las partes más altas hay bosques secundarios de hoja ancha y una porción de bosque latifoliado maduro. Basado en la cobertura vegetal y en el grado 
Agudelo et al.: Extensión del Bosque Nublado y su Contribución de Lluvia Horizontal a la Precipitación Total

de epifitismo, el límite altitudinal de la zona impactada por nubes o neblinas existe actualmente a partir de los $1,600 \mathrm{~m}$ de elevación (Figura 4). Esto es $100 \mathrm{msnm}$ más abajo del límite actual del área núcleo de la RBU.

La zona núcleo de la RBU fue delimitada a partir de los $1,700 \mathrm{msnm}$, un área de 237.1 ha (Mora et al. 2013). Los levantamientos de campo en este estudio indican que de esta superficie, unas 52.0 ha están cubiertas por el bosque latifoliado maduro y el resto por bosques secundarios de coníferas y latifoliadas. De igual manera, determinamos que el límite de la zona impactada por las nubes o las neblinas está a unos 1,600 msnm (Figura 4). Esto es un área de 322.0 ha que, sumadas a las 237.1 ha actualmente delimitadas como zona núcleo, constituyen una superficie de 559.0 ha que equivalen a la zona de recarga hídrica del cerro Uyuca. Las 322.0 ha entre los 1,600 y los 1,700 msnm están cubiertas principalmente por pinabete. No obstante, 157.0 ha $(48 \%)$ no están dentro de los límites de la RBU. A la vez, parte de las 165.0 ha que están dentro de los límites de la RBU está intervenida por la agricultura (Figura 4).

Debido a su valor en la captación y regulación del agua, todas las 322.0 ha, o al menos los sectores más factibles, deben ser preservadas estrictamente para proteger un área de bosque nublado de gran relevancia. Esto porque aparte de los bienes y servicios ecosistémicos que esta área aporta, su contribución de lluvia horizontal es primordial para el balance hídrico de la montaña y por lo tanto para la provisión de agua a las comunidades demandantes al pie de monte del cerro Uyuca. Debido a esta razones, Mora y López (2011a) y Mora et al. (2013) enfatizaron en la demarcación y delimitación como parte de la zonificación de la RBU de una zona de manejo especial en la zona de amortiguamiento adyacente a la zona núcleo. Esta Subzona de Adaptación al Cambio Climático está delimitada entre la cota de los 1,600 msnm y la cota de los $1,700 \mathrm{msnm}$ del cerro (Figura 4). La Subzona de Adaptación al Cambio Climático incluye sectores de bosque mixto ralo, bosque de pino en regeneración, bosque de pino ralo, bosque en regeneración y, principalmente, bosque de pino denso.

Desde el punto de vista hidrológico, toda la zona de recarga del cerro Uyuca debería estar totalmente cubierta con bosque latifoliado maduro multiestratificado. Esta cobertura vegetal garantizaría que la precipitación total (precipitación vertical y horizontal) fuese superior a los $2,360 \mathrm{~mm}$, con un aporte de casi el $150 \%$ de la precipitación horizontal sobre la precipitación vertical. Es posible que con el actual tipo de cobertura de bosque secundario de la RBU, se estén perdiendo grandes cantidades de vapor de agua que podrían ser incorporadas al suelo por procesos de condensación. Tales pérdidas se traducirían en deficiencias hídricas en las partes bajas de la montaña. A esto hay que añadirle que la altura de la capa de nubes durante la estación seca se ha estado elevando a una tasa de $2 \mathrm{~m}$ por año en varios sitios. Esto se debe al menos parcialmente a la deforestación (Ramirez 2011).

Es común que las áreas deforestadas terminen como pastizales. Esto es un problema debido a que se espera que a largo plazo el agua superficial y subsuperficial que alimenta el caudal base de los ríos sea mayor en los bosques que en los pastizales (Ramirez 2011). La precipitación oculta que se da dentro del bosque latifoliado maduro de la RBU es un aporte importante a la precipitación normal y por lo tanto para todas las comunidades que la utilizan, incluido la Escuela Agrícola Panamericana. Esto por cuanto el actual suministro de agua potable y de riego para los usos en la Escuela Agrícola Panamericana se deriva casi exclusivamente del cerro Uyuca.

Debido a todo lo anterior, se debe levantar un mapa de las etapas serales o sucesionales de toda la RBU. Esto porque últimamente se ha detectado una pequeña reducción de la masa de bosque latifoliado maduro. Este mapa se debería utilizar como instrumento de partida para reiniciar algunos esquemas de restauración de la masa secundaria. Completado este estudio, se debería comenzar un proyecto de restauración ecológica con propósitos hidrológicos y de biodiversidad. Esto es fundamental, sobre todo si se tiene en cuenta que debido a su delicada dependencia del clima local, los bosques nubosos serán fuertemente afectados por el cambio climático global (Bruinjnzeel y Hamilton 2000).

La escorrentía y sus componentes son controlados por los factores climáticos y las propiedades del paisaje (Xu et al. 2012). El patrón espacial de la cobertura de la vegetación es en respuesta a la disponibilidad de agua (Caylor et al. 2005). Se ha determinado que la cobertura de vegetación leñosa total se satura con un nivel de precipitación de 600$1000 \mathrm{~mm}$ en todos los ecosistemas de la sabana africana. El cambio climático global cambiará el régimen de escorrentía (Barnett et al. 2005) y sus extremos, así como la productividad de la vegetación (Knapp y Smith 2001). Debido a que el crecimiento de 
la vegetación es afectado por la intermitencia de la disponibilidad de agua (Baudena et al. 2007), cualquier cambio espacial y temporal de la precipitación se esperaría que influya mucho sobre la variabilidad de la cobertura vegetal (Xu et al. 2012). Los hidrólogos han puesto mucha atención a averiguar qué cantidad del cambio observado en los componentes del equilibrio hídrico (escorrentía y sus componentes) y la cobertura vegetal (leñosa y no leñosa) puede ser atribuida a la variabilidad del clima (Xu et al. 2012).

Se necesita conocer el estado de conservación de cada tipo de bosque de la RBU para trabajar en los aspectos de manejo que requieran. Se debe aumentar el área de bosque latifoliado multiestratificado maduro como posible amortiguador del efecto negativo del cambio climático global. En la Subzona de Adaptación al Cambio Climático se podrá experimentar y manipular el hábitat mediante el manejo experimental. Este manejo incluye la regeneración asistida, la restauración ecológica y otras técnicas para mejorar la cobertura boscosa con fines hídricos, de biodiversidad y de conservación. La Subzona de Adaptación al Cambio Climático ocupa una superficie de 164.84 ha dentro de la RBU (Mora et al. 2013).

Debido al efecto del huracán Hugo en 1989, la línea de nubes subió $300 \mathrm{~m}$ en las montañas Luquillo en Puerto Rico debido a la defoliación de los árboles de las laderas de barlovento de la montaña. No obstante, el efecto desapareció algunos meses después de que las hojas crecieron nuevamente en los árboles (Bruinjnzeel y Hamilton 2000). La Subzona de Adaptación al Cambio Climático de Uyuca deberá cumplir un papel primordial en el mantenimiento de las condiciones climáticas del cerro Uyuca.

Cada vez existe más evidencia científica para la elevación de la base de nubes asociada con el calentamiento global. En las montañas de picos múltiples, a este efecto se le debe adicionar el aumento de la fragmentación del hábitat, lo que disminuiría aún más las probabilidades de supervivencia de la biodiversidad remanente (Bruinjnzeel y Hamilton 2000). El efecto asociado a esta elevación de la línea de nubes puede tener consecuencias muy serias para la biodiversidad.

En un área de $30 \mathrm{~km}^{2}$ en Monteverde, Costa Rica, se determinó una asociación entre el calentamiento reciente y la extinción masiva de 20 especies de anuros de las 50 que existían (Pounds et al. 1999). Varias especies de fauna están variando su ámbito de distribución, probablemente debido al efecto compuesto de la deforestación, el cambio climático y algunos otros factores de afectación a la biodiversidad (Mora y López 2011b). Los procesos de adaptación al cambio climático en Uyuca deben incluir medidas como el enriquecimiento de la masa forestal latifoliada. No obstante, es poco lo que se sabe sobre el efecto de la forma y el tamaño de las hojas sobre la intercepción horizontal. Algunos consideran que las coníferas son más eficientes que las latifoliadas en esa actividad (Vogelmann 1973). Este último autor determinó que la neblina fue interceptada eficientemente por las copas de los árboles al pasar por un grupo aislado de pinos en Las Vigas, México, lo que resultó en gotas de agua que cayeron de las acículas y saturaron el suelo hasta una profundidad de 8 a $10 \mathrm{~cm}$.

Hay varios otros temas de investigación que son prioritarios en la RBU, desde el punto de vista hidrológico y de las perspectivas de su persistencia ante los eminentes efectos del cambio climático global. Entender las interacciones entre el clima, la vegetación y el balance hídrico, especialmente en las áreas con poca precipitación, al menos estacionalmente, es uno de los temas principales de estudio en la ecohidrología de las cuencas (Xu et al. 2012). Por lo tanto, se deben continuar los estudios para afinar los resultados de esta investigación y evaluar el impacto hidrológico de las grandes masas de pinabete en la RBU así como en la Subzona de Adaptación al Cambio Climático. Se debe estudiar en detalle la eficiencia de la captación de la niebla por la vegetación para determinar con más precisión la intercepción (García-Santos et al. 2004). La cantidad de lluvia horizontal puede ser medida con más precisión con el análisis de los isótopos en el agua (Santiago et al. 2005). De igual manera, el contenido preciso de agua en el suelo debe ser medido a través de perfiles en puntos representativos espacialmente. También se debe estudiar en planos de flujo cero la recarga de las aguas freáticas.

\section{Literatura Citada}

Aparicio, F.J. 2006. Fundamentos de hidrología de superficie. Limusa, México, D.F. 302 p.

Barnett, T.P., J.C. Adam y D.P. Lettenmaier. 2005. Potential impacts of a warming climate on water availability in snow-dominated regions. Nature 438(7066):303-309.

Baudena, M., G. Boni, L. Ferraris, J. von Hardenberg y A. Probenzale. 2007. Vegetation response to rainfall intermittency in drylands: Results from a simple ecohydrological box model. Advances in Water Resources 30(5):1320-1328. 
Bubb, P., M. Aldrich y J. Sayer. 2002. Los bosques de niebla tropicales de montaña: es hora de actuar. UNASYLVA 53(208): 36-37.

Bruijnzeel, L. A. y L.S. Hamilton. 2000. Decision time for cloud forests, Water related issues and problems of humid tropics and other warm humid regions, WWF, IHP, IUCN. IHP Humid Tropics Programme Series No. 13. UNESCO. $44 \mathrm{p}$.

Cáceres, G. 1981. Importancia hidrológica de la intercepción horizontal en un bosque muy húmedo premontano en Balalaika, Turrialba, Costa Rica. Tesis de posgrado en Ciencias Agrícolas y Recursos Naturales, CATIE/ Universidad de Costa Rica. Turrialba, Costa Rica. 98 p.

Cavelier, J. y G. Goldstein. 1989. Mist and fog interception in elfin cloud forests in Colombia and Venezuela. Journal of Tropical Ecology 5(03):309-322.

Caylor, K.K., S. Manfreda e I. Rodriguez-Iturbe. 2005. On the coupled geomorphological and ecohydrological organization of river basins. Advances in Water Resources 28(1):69-86.

Chow, V., D. Maidment y L. Mays. 1994. Hidrología Aplicada. Mc Graw-Hill. Bogotá, Colombia. 583 p.

García-Santos, G., M.V. Marzol y G. Aschan. 2004. Water dynamics in a laurel montane cloud forest in the Garajonay national park (Canary Islands, Spain). Hydrology and Earth Systems Sciences 8(6):1065-1075.

Kappelle, M. y A.D. Brown. 2001. Bosques nublados del neotrópico. INBio, Heredia, Costa Rica. 698 p.

Knapp, A.K. y M.D. Smith. 2001. Variation among biomass in temporal dynamics of aboveground net primary production. Science 291(5503):481-484.

Liniger, H. y R. Weingartner. 1998. Montañas y recursos hídricos. UNASYLVA 49 (195): 39-46.

Mora, J.M y L.I. López. 2011a. El Manejo de la Reserva Biológica Uyuca en el Contexto Nacional y Global del Sistema de Áreas Protegidas. Ceiba 52(1):39-54.

Mora, J.M. y L.I. López. 2011b. Cambios en la Distribución de las Aves: ¿Qué Puerta Hemos Abierto?; los Casos del Chico Piojo (Campylurhynchus rufinucha) y la Paloma Ala Blanca (Zenaida asiatica) en Costa Rica. Ceiba 52(2):230-236.

Mora, J.M., L.I. López, M. Acosta y P. Maradiaga. 2013. Plan de Manejo Reserva Biológica Uyuca 2013-2025. Instituto Nacional de Conservación y Desarrollo Forestal, Áreas Protegidas y Vida Silvestre y Escuela Agrícola Panamericana. Honduras. 165 p.

Ponce-Reyes, R., V.H. Reynoso-Rosales, J.E.M. Watson, J. VanDerWal, R.A. Fuller, R.L. Pressey y H.P. Possingham. 2012. Vulnerability of cloud forest reserves in Mexico to climate change. Nature Climate Change 2(6):448-452.
Pounds, J.A., M.P.L. Fogden y J.H. Campbell. 1999. Biological response to climate change on a tropical mountain. Nature 398(6728):611-615

Pratt, J. y L. Preston. 1998. Economía del flujo de los recursos de montaña. UNASYLVA 49(4):31-38

Price, M.F. 1998. Las montañas: ecosistemas de importancia mundial. UNASYLVA 49 (4):3-12.

Ramírez, A.I. 2011. Contribución hidrológica de la precipitación horizontal en un bosque nublado de la zona del Trifinio, América Central. Tesis Magister Scientiae en Manejo y Gestión Integral de Cuencas Hidrográficas, CATIE, Costa Rica. 87 p.

Santiago, L.S., K. Silvera, J.L. Andrade y T.E. Dawson. 2005. El uso de isótopos estables en biología tropical. INCI, Caracas, v. 30, n. 9. Disponible en http://www.scielo.org.ve/scielo.php?script=sci_arttext\&pi $d=S 0378-18442005000900006 \& \operatorname{lng}=e s \& n r m=i s o$

Singleton, F. 2008. The beaufort scale of winds "its relevance, and its use by sailors" (en línea). Weather 63 (2):37-4. Consultado 24 de marzo de 2015. Disponible en http://onlinelibrary.wiley.com/doi/10.1002/wea.153/abstr act

Stadtmüller, T. 1987. Los bosques nublados en el trópico húmedo. Universidad de las Naciones Unidas y Centro Agronómico Tropical de Investigación y Enseñanza. Turrialba, Costa Rica. 85 p.

Stadtmüller, T. y N. Agudelo. 1990. Amount and variability of cloud moisture input in a tropical cloud forest. International Association of Hydrological Sciences Publication 193:25-32.

Still, C.J., P.N. Foster y S.H. Schneider. 1999. Simulating the effects of climate change on tropical montane cloud forests. Nature 398(6728):608-610

Vargas, G. 2006. Geografía de Costa Rica. EUNED. San José, Costa Rica. 265 p.

Vogelmann, H. 1973. Fog Precipitation in the Cloud Forest of Eastern Mexico. BioScience 23(23): 96-100.

Wadsworth, F.H. 2000. Producción forestal para América Latina. IUFRO-SPDC Textbook Project no.3. CATIE, IUFRO, Servicio Forestal, Departamento de Agricultura de los EEUU. 603 p.

Waechter, J.L. 1998. Epifitismo vascular em uma floresta de restinga do Brasil subtropical. Ciência e Natura 20:4366.

Xu, X.D. Yang y M. Sivapalan. 2012. Assessing the impact of climate variability on catchment water balance and vegetation cover. Hydrology and Earth System Sciences 16(1): 43-58,

Recibido para publicación el 22 de marzo de 2015. Aceptado para publicación el 21 de noviembre de 2015. 